\title{
Ars2 : un nouveau régulateur central de l'identité des cellules souches neurales
}

Celia Andreu-Agullo, Thomas Maurin

Facteurs de transcription et cellules souches neurales

Les cellules souches adultes ont la double propriété de proliférer à l'infini sans perte de potentiel, c'est-à-dire de s'autorenouveler et de pouvoir se différencier en un ou plusieurs types cellulaires. Ces deux caractères confèrent leur identité aux cellules souches. Un des principaux enjeux de la recherche sur ce type cellulaire singulier est de comprendre les mécanismes moléculaires qui permettent le maintien de leur identité [1].

Les progrès techniques ont permis de mettre un terme au dogme selon lequel les neurones sont exclusivement produits au cours du développement. Dès 1960, des études d'incorporation de thymidine tritiée dans les cerveaux de rats adultes ont permis de démontrer la formation de nouveaux neurones dans différentes régions du cerveau. Plus tard, des cellules souches neurales (CSN) ont été identifiées. La quasiquiescence de ces cellules permet de les suivre grâce à leur incorporation à long terme d'analogues nucléotidiques dérivés du BrdU (bromodéoxyuridine). Ainsi, on distingue dans le système nerveux central adulte deux zones neurogènes principales hébergeant ces CSN, I'hippocampe et la zone sous-ventriculaire (SVZ) [2] (Figure 1).

Les facteurs de transcription jouent un rôle central dans la physiologie des cellules souches. Certains contrôlent leur multiplication quand d'autres orchestrent leur différenciation [3]. Ainsi, plusieurs protagonistes interviennent de façon cruciale pour contrôler ces processus au niveau des CSN. C'est le cas de la voie de signalisation en aval des récepteurs Notch. En effet, les cellules qui expriment fortement CBFl (ou RBPJk) - un facteur de transcription ubiquitaire médiateur nucléaire de l'action de Notch - ont une plus grande capacité à s'autorenouveler que celles qui l'expriment faiblement et qui, elles, sont déjà engagées dans leur différenciation [4]. Par ailleurs, l'autorenouvellement des CSN issues de souris doublement invalidées

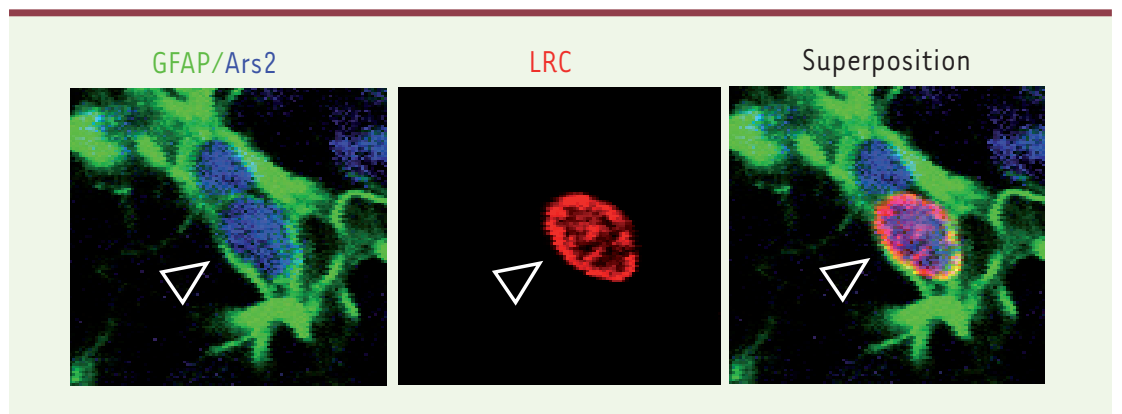

Figure 1. Ars2 est exprimée par les CSN. Marquage immunohistochimique d'une section coronale du cerveau d'une souris transgénique adulte GFAP-GFP. GFAP (vert), Ars2 (bleu) et CldU (rouge). LRC : label retaining cell.
Department of developmental biology, Sloan-Kettering institute, 1275 York Avenue, Box 252, New York, Ny 10065, États-Unis. thomas.maurin@gmail.com

pour les gènes Hesl (de la famille des hairy enhancer of split, gènes cibles de Notch) et Hes5 est réduit. Le récepteur nucléaire orphelin Tlx est lui aussi nécessaire au maintien de l'état indifférencié des CSN. En effet, chez les souris adultes qui en sont dépourvues, la prolifération cellulaire et l'expression de la nestine dans les zones neurogènes sont diminuées [5]. Bmi, HMGA2 (high mobility group AT-hook 2), ou encore Gli (facteur de transcription à doigts de zinc) interviennent également dans le contrôle de l'autorenouvellement des CSN [3]. Quant aux membres de la famille SoxB1 (Soxl, Sox2 et Sox3), les CSN les expriment tout au long du développement et jusqu'à l'âge adulte. Un défaut d'expression de Sox2 et ou Sox3 dans les précurseurs neuronaux entraîne leur sortie prématurée du cycle cellulaire et un début de différenciation [6]. À l'inverse, la surexpression de Sox2 inhibe la différenciation neuronale et force les CSN à conserver leur caractère indifférencié [7]. Enfin, Sox2 est essentiel au maintien des CSN dans les zones neurogènes chez l'adulte $[8,9]$.

Les cellules souches adultes et les progéniteurs qu'elles engendrent sont identifiables non seulement par leur morphologie ou leur localisation, mais aussi grâce à un certain nombre de marqueurs spécifiques (Tableau / et Figure 1). Grâce aux techniques d'imagerie, d'ARN interférence et de génétique, nous avons caractérisé le rôle clé de Ars2 (arsenite-resistance protein 2, aussi connue sous le nom de Srrt) dans le maintien de l'identité souche des CSN [10]. 


\begin{tabular}{|c|c|c|c|c|}
\hline Type cellulaire & $\begin{array}{l}\text { Nomen- } \\
\text { clature }\end{array}$ & Marqueurs endogènes & $\begin{array}{c}\text { Marquage } \\
\text { à long terme (LRC) }\end{array}$ & $\begin{array}{c}\text { Expression } \\
\text { Ars } 2\end{array}$ \\
\hline Cellules souches neurales & B & $\begin{array}{l}\text { GFAP }^{+} \text {Sox } 2^{+} / \mathrm{GFAP}^{+} \text {Lex }^{+} / \mathrm{GFAP}^{+} \\
\text {Prominin }\end{array}$ & oui & oui \\
\hline Transit amplifying progenitors (TAP) & c & Mashl $^{+}$ & non & oui \\
\hline Neuroblastes (NB) & A & $\mathrm{DCX} X^{+}$ & non & non \\
\hline Astrocytes matures et parenchymateux & & $\mathrm{GFAP}^{+} \mathrm{S} 100 ß^{+}$ & non & non \\
\hline Cellules astrogliales (non pluripotentes) & & GFAP $^{+}$Sox2 $2^{-} /$GFAP $^{+}$Nestine $^{-}$ & non & non \\
\hline Cellules de l'épendyme & $\varepsilon$ & $\mathrm{S} 100 \beta^{+}$ & non & oui \\
\hline $\begin{array}{l}\text { Interneurones granuleux } \\
\text { et périglomérulaires }\end{array}$ & & $\begin{array}{l}\text { Tyrosine hydroxylase }(\mathrm{TH}) \text {, } \\
\text { calretinin (CR) ou calbindin } \\
\text { (CB) }\end{array}$ & oui & nd \\
\hline
\end{tabular}

Tableau I. Récapitulation des marqueurs endogènes permettant l'identification des différentes cellules filles des CSN. nd : non déterminé.

\section{Ars2 est exprimée par les cellules} souches adultes neurales

$\varepsilon n$ réalisant des comarquages en immunohistochimie (Figure 1), nous avons démontré que Ars2 est exprimée dans les cellules souches neurales (coexprimant GFAP [glial fibrillary acidic protein] et CD133). Ainsi, $90 \%$ des cellules GFAP $^{+}$incorporant à long terme le CldU (5-chloro-2'-déoxyuridine), et nommées LRC pour label retaining cells, coexpriment Ars2. En revanche, Ars2 est quasi absente des populations de cellules ayant engagé leur différenciation. Par exemple, dans les TAP (transit amplifying progenitors) exprimant Mashl, l'expression d'Ars2 est faible (seulement $7 \%$ des TAP expriment Ars2), et elle est quasi nulle dans les neuroblastes (exprimant Dcx, doublecortine), les cellules astrogliales (GFAP ${ }^{+} / \mathrm{Nes}^{-}$ tine ${ }^{-} / \mathrm{Sox}^{-}$) et les astrocytes matures $\left(\mathrm{S} 100 \mathrm{~b}^{+}\right)$. L'expression d'Ars2 décroît donc au cours de l'engagement des lignées issues de la SVZ, ce qui suggère que cette molécule peut jouer un rôle dans le maintien du caractère souche des CSN.

\section{Quel rôle pour Ars2 in vivo ?}

Nous avons cherché à diminuer l'expression d'Ars2 dans les CSN grâce à la technique d'ARN interférence tout en suivant le devenir de ces cellules.
Des shARN spécifiques de la version murine d'Ars2 ont été clonés dans des vecteurs lentiviraux. Ces vecteurs ont été pseudotypés avec la glycoprotéine d'enveloppe du virus Mokola qui permet de n'infecter que les cellules de la lignée astrogliale. En utilisant un cadre stéréotaxique, nous avons injecté ces virus dans la SVZ, au plus près des CSN (Figure 2). Nous avons ensuite réalisé des marquages en immunohistochimie et étudié le devenir des cellules infectées (exprimant la GFP) chez les souris injectées avec les lentivirus soit

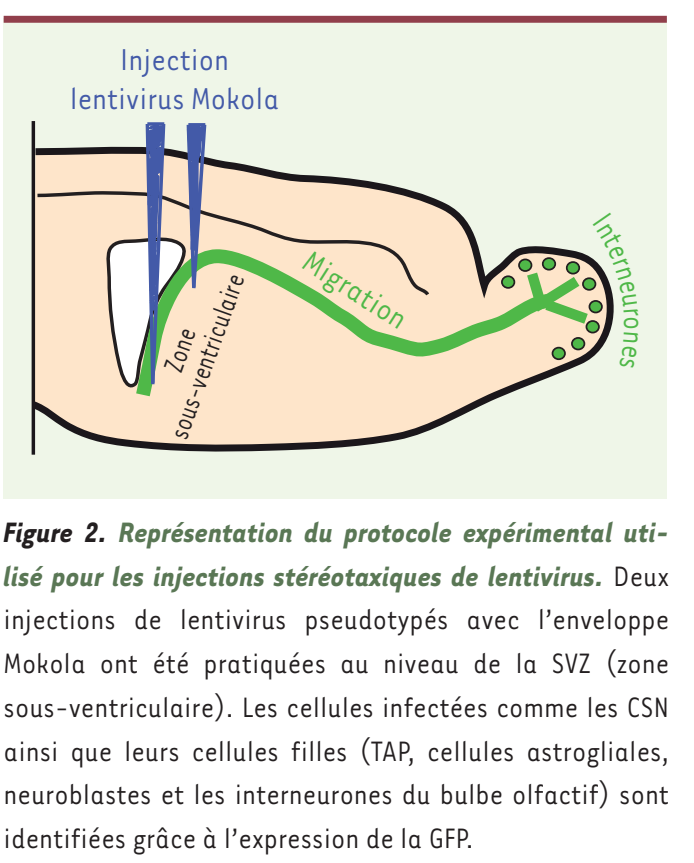

contrôles (shT), soit codant le shARN ciblant Ars2 (shArs2). Chez ces dernières, nous avons observé une forte réduction du nombre des cellules souches neurales $\mathrm{GFAP}^{+} /$Nestine $^{+}$et des neuroblastes $D C X^{+}$. En accord avec des travaux antérieurs expliquant la perte des cellules souches par leur engagement dans la différenciation astrocytaire $[5,11]$, nous avons observé que le nombre d'astrocytes matures - reconnus par l'expression de $5100 \beta^{+}$- infectés était augmenté de $50 \%$ chez les souris ayant reçu les vecteurs shArs2. Enfin, un mois après l'injection des lentivirus, le nombre d'interneurones néoformés dans le bulbe olfactif est fortement diminué chez les souris traitées avec le shArs2. L'autorenouvellement est une propriété difficile à analyser car il faut démontrer qu'il y a eu division cellulaire tout en s'assurant que les cellules filles conservent leur potentiel de différenciation. On peut cultiver les CSN in vitro sous forme de sphères non adhérentes, les « neurosphères ». Ainsi, compter le nombre de nouvelles neurosphères générées à chaque passage en culture 
est une mesure fiable de l'autorenouvellement. Nous avons confirmé nos observations faites in vivo en cultivant les CSN issues des souris traitées par les shArs2. Ces cellules n'étaient plus capables de produire de neurosphères, indiquant un défaut d'autorenouvellement en absence d'Ars2. À l'opposé, la surexpression d'Ars2 entraîne une augmentation significative du nombre de neurosphères formées, ce qui suggère que Ars2 est nécessaire et suffisante pour le maintien de l'autorenouvellement des CSN chez l'adulte.

Nous avons cherché à approfondir nos résultats en invalidant de façon conditionnelle le gène Ars2 (Ars2 cKO). Pour cela, nous avons croisé des souris Ars $2^{f l / f l}$ avec des souris hGFAP-cre. Le promoteur hGFAP permet l'expression de cre à partir du jour embryonnaire $\varepsilon 13,5$, ce qui entraîne donc la suppression de l'expression d'Ars2 dans les cellules de la lignée astrogliale. Les souris Ars2 ckO sont nées avec la distribution mendélienne attendue et sans aucun phénotype notable à la naissance. Cependant les mutants développent progressivement un retard de croissance, une ataxie et une hydrocéphalie et meurent environ 20 jours après leur naissance. Les coupes histologiques des cerveaux des souris Ars 2 cKO réalisées 15 jours après leur naissance montrent un élargissement des ventricules et une diminution de la taille des bulbes olfactifs. Dans la SVZ, le nombre de CSN (de phénotype Nestine ${ }^{+} /$Sox $^{+} /$LeX $^{+} /$GFAP $^{+}$) est effondré (< $20 \%$ du nombre observé chez les contrôles), et celui des cellules de la lignée astrogliale (GFAP ${ }^{+} \mathrm{S} 100 \mathrm{~b}^{+}$) fortement augmenté. Ces observations confirment donc le rôle d'Ars2 dans le maintien des CSN et dans le contrôle de la neurogenèse postnatale.

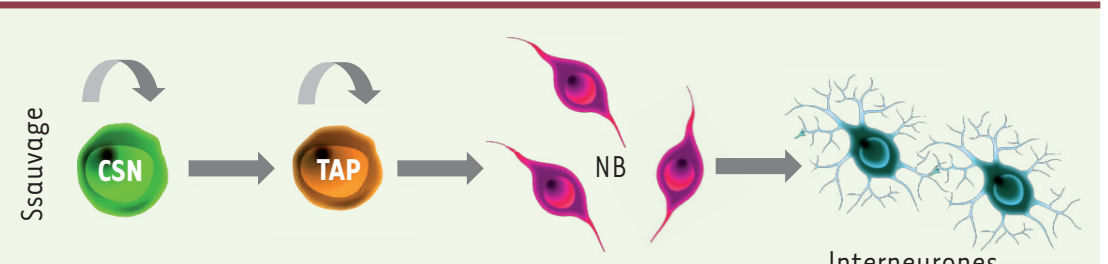

Surexpression de Sox2

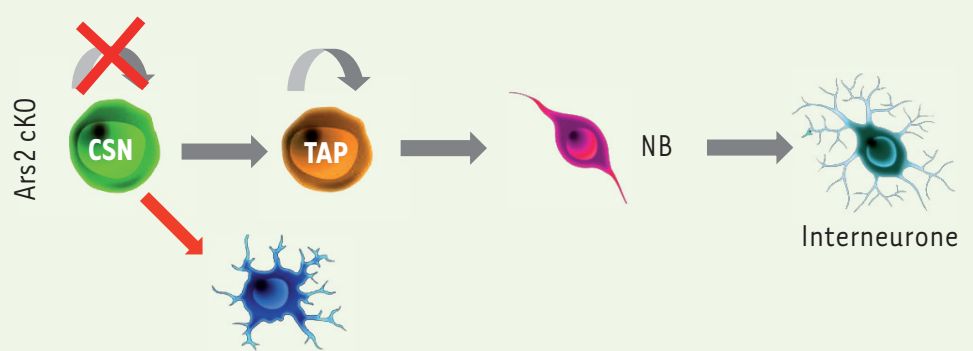

Astrocyte non pluripotent

Figure 3. Ars2 est un régulateur central de l'identité des CSN. Chez les souris sauvages, les CSN (qui expriment Ars2) sont capables de s'autorenouveler et de générer des neuroblastes (NB) qui migrent vers le bulbe olfactif où ils se différencient en interneurones. En l'absence d'Ars2, les CSN sont dans l'incapacité d'assurer la neurogenèse et se différencient en astrocytes. Cette diminution du compartiment des CSN explique que les souris invalidées pour Ars2 génèrent moins de nouveaux interneurones. La surexpression de Sox2 compense ce phénotype en restaurant la pluripotence et l'autorenouvellement des CSN chez les souris Ars2 cKO.
Quels sont les facteurs de transcription responsables des effets d'Ars2 ?

Étant donné le rôle majeur de Hesl, Hes5 et Sox2 dans le maintien des CSN, nous avons analysé leurs niveaux d'expression dans les cellules transduites avec les lentivirus shArs2 et shT. Les niveaux de Sox2 sont drastiquement diminués lorsque l'expression d'Ars2 est altérée. Des expériences d'immunoprécipitation de la chromatine nous ont permis de montrer que Ars2 se lie à une région spécifique du promoteur de Sox2. Ars2 active la transcription de Sox2 dans les CSN, mais cette fonction pourrait être spécifique du contexte cellulaire. En effet, Sox2 n'est pas activé dans les cellules non neuronales 3T3 qui, pourtant, expriment de forts niveaux d'Ars2.

Afin de valider ce rôle d'effecteur clé d'Ars2, nous avons transfecté in utero un vecteur permettant la surexpression de Sox2 dans le cerveau des embryons Ars2 cKO. Comme attendu, toutes les capacités d'autorenouvellement et de pluripotence des CSN issues de ces souris ont été restaurées. Ce faisceau d'observations, ajouté aux résultats de nos expériences in vivo, établit Ars2 comme un nouveau régulateur central de l'expression de Sox2.

Ars2 avait été précédemment décrite pour son rôle dans la résistance à l'arsenic, puis plus tard dans la biogenèse des microARN et plus généralement dans les mécanismes moléculaires de l'ARN interférence [12, 13]. Dans le cadre de notre étude, nous avons découvert un nouveau rôle pour Ars2, totalement indépendant de ces autres fonctions décrites précédemment. En effet, nous démontrons que Ars2 est exprimée dans les cellules souches neurales et que sa présence décroît au cours de leur différenciation. Nous montrons que Ars2 est une protéine qui lie spécifiquement l'ADN et qu'elle est un régulateur majeur de l'expression de Sox2. Ainsi, Ars2 est un nouveau partenaire moléculaire essentiel au maintien de l'identité des cellules souches neurales (Figure 3). 


\section{Perspectives}

Notre étude soulève de nouvelles interrogations dont certaines sont actuellement au centre des recherches menées dans le laboratoire d'Eric Lai. Par exemple, quel est le rôle d'Ars2 dans l'autorenouvellement des cellules souches embryonnaires ? En effet, le développement embryonnaire des souris invalidées pour Ars2 est bloqué à un stade précoce qui rappelle fortement le phénotype des souris invalidées pour Sox2. Par ailleurs, il y a une ressemblance frappante entre les cerveaux des souris ayant une invalidation conditionnelle d'Ars2 et ceux des souris porteuses d'un mutant Sox hypomorphe [8]. Cependant, les cerveaux des souris mutantes hypomorphes Sox2 ne présentent pas d'astrogliose majeure comme ceux des souris Ars2. Il se pourrait donc que Ars2 contrôle la différenciation vers la lignée astrogliale de manière spécifique, en contrôlant simultanément des gènes autres que Sox2. Des travaux d'immunoprécipitation de la chromatine couplée à du séquençage à haut débit sont en cours afin de caractériser de nouveaux gènes cibles d'Ars2.
Identifier ces gènes pourrait s'avérer très utile pour décrypter la fonction de cette molécule, compléter notre compréhension du phénotype des souris Ars2 cKO et, à plus long terme, imaginer de nouvelles perspectives thérapeutiques. En effet, peut-être l'activation de l'expression d'Ars2 dans certaines tumeurs cérébrales comme les astrogliomes pourrait-elle permettre de réprimer la prolifération des cellules cancéreuses. $\diamond$ Ars2, an essential player in neural stem cell identity

\section{LIENS D'INTÉRÊT}

Les auteurs déclarent n'avoir aucun lien d'intérêt concernant les données publiées dans cet article.

\section{REMERCIEMENTS}

Nous remercions D. Pisani, L. Boyer et B. Mari pour leur relecture critique de ce manuscrit. Nous remercions tout spécialement Eric C. Lai pour son soutien et pour nous avoir donné tous les moyens nécessaires pour réaliser cette étude.

\section{RÉFÉRENCES}

1. Shi Y, Sun G, Zhao C, Stewart R. Neural stem cell selfrenewal. Crit Rev Oncol Hematol 2008 ; 65 : 43-53.

2. Doetsch $F$. The glial identity of neural stem cells. Nat Neurosci $2003 ; 6: 1127-34$
3. Ahmed S, Gan HT, Lam CS, et al. Transcription factors and neural stem cell self-renewal, growth and differentiation. Cell Adh Migr 2009 ; 3 : 412-24.

4. Mizutani K, Yoon K, Dang L, et al. Differential Notch signalling distinguishes neural stem cells from intermediate progenitors. Nature 2007 ; 449 : 351-5.

5. Shi Y, Chichung Lie D, Taupin P, et al. Expression and function of orphan nuclear receptor TLX in adult neural stem cells. Nature $2004 ; 427: 78-83$.

6. Graham V, Khudyakov J, Ellis P, Pevny L. SOX2 functions to maintain neural progenitor identity. Neuron 2003 ; $39: 749-65$.

7. PevnyL, Placzek M. SOX genes and neural progenitor identity. Curr Opin Neurobiol 2005 ; 15 : 7-13.

8. Ferri AL, Cavallaro M, Braida D, et al. Sox2 deficiency causes neurodegeneration and impaired neurogenesis in the adult mouse brain. Development 2004 ; 131 : 3805-19.

9. Suh $\mathrm{H}$, Consiglio A, Ray J, et al. In vivo fate analysis reveals the multipotent and self-renewal capacities of Sox $2^{+}$neural stem cells in the adult hippocampus. Cell Stem Cell $2007 ; 1: 515-28$.

10. Andreu-Agullo C, Maurin T, Thompson CB, Lai $\varepsilon$. C. Ars2 maintains neural stem-cell identity through direct transcriptional activation of Sox2. Nature 2012 ; 481 195-8.

11. Raponi $\varepsilon$, Agenes F, Delphin C, et al. S100B expression defines a state in which GFAP-expressing cells lose their neural stem cell potential and acquire a more mature developmental stage. Glia 2007 ; 55 : $165-77$.

12. Sabin LR, Zhou R, Gruber JJ, et al. Ars2 regulates both miRNA- and siRNA-dependent silencing and suppresses RNA virus infection in Drosophila. Cell $2009 ; 138: 340-51$.

13. Yang L, Liu Z, Lu F, et al. Serrate is a novel nuclear regulator in primary microRNA processing in Arabidopsis. Plant J $2006 ; 47: 841-50$

\section{NOUVELLE}

\section{Le paludisme : quelle place pour l'axe hepcidine-fer?}

Sophie Vaulont ${ }^{1}$, Laurent Rénia ${ }^{2}$, Dominique Labie ${ }^{1}$
${ }^{1}$ Institut Cochin, Inserm U1016, CNRS UMR 8104, 24, rue du Faubourg Saint-Jacques, 75014 Paris, France;

${ }^{2}$ Singapore immunology network (SIgN), 8A biomedical grove, immunos building, level 4, Singapore.

sophie.vaulont@inserm.fr
> Le fer fait partie des nutriments les plus convoités par les pathogènes pour se multiplier. Ces derniers ont de fait développé des systèmes sophistiqués pour récupérer de façon maximale, parfois aux dépens de l'hôte, ce métal si précieux [1]. Au cœur de cette bataille, l'hepcidine joue un rôle important dans la défense de l'hôte. L'hepcidine est une hormone hyposidérémiante, induite lors d'épisodes infectieux, qui agit en limitant l'export du fer des entérocytes (provenant du fer alimentaire) et des macrophages (provenant du catabolisme de l'hémoglobine) [2]. Les données récentes de la littérature sur le paludisme proposent un bel exemple de la place importante tenue par l'axe hepcidine-fer dans la compétition hôte-pathogène.

\section{Physiopathologie du paludisme}

Le paludisme, malaria dans la littérature anglophone, est une des maladies infectieuses les plus fréquentes au monde, responsable chaque année de plus d'un million de décès, transmise par la piqûre d'un moustique femelle infecté [3]. Cette piqûre introduit dans l'organisme l'agent infectieux, le Plasmodium. En Afrique subsaharienne, l'agent infectieux dominant est le Plasmodium falciparum et le principal vecteur l'Anopheles gambiae. Introduit au niveau de la peau, le sporozoïte gagne la circulation et les sinusoïdes hépatiques. Il parvient ainsi aux hépatocytes, il y forme une vacuole parasitophore où 